\title{
Can embryo metabolism be used for selecting bovine embryos before transfer?
}

\author{
Isabelle Donnaya* Robert J. Partridge ${ }^{\mathrm{b}}$, Henry J. Leese ${ }^{\mathrm{b}}$ \\ ${ }^{a}$ Unité vétérinaire, Université catholique de Louvain, Place Croix du Sud 3, B-1348, \\ Louvain-La-Neuve, Belgium \\ ${ }^{b}$ Department of Biology, University of York, York, UK
}

(Received 21 June 1999; accepted 12 July 1999)

\begin{abstract}
The quality of in vitro-produced bovine embryos remains variable. The selection of these embryos based only on their morphology does not allow for acceptable gestational rates to be obtained. The use of metabolic markers to select viable embryos before transfer would be of valuable help, both economically and as a research tool. The ideal marker should meet several conditions: it should be able to be evaluated 1) in a totally non-invasive manner, 2) on individual embryos (which necessitates very sensitive techniques), 3 ) very rapidly (so that it is compatible with the immediate transfer of fresh embryos), and 4) in order to allow viable embryos to be separated from those that are not viable, whatever the production system used. In practice, such a marker does not exist, but certain methods of metabolic evaluation resemble it. The development of a metabolic marker is confronted by the metabolic characteristics of the embryo, notably the evolution of the metabolism during the development of the embryo and its adaptation to the changes in the environment. $\mathcal{C}$ Inra/Elsevier, Paris
\end{abstract}

metabolism / embryo / cattle / marker / in vitro

Résumé - Peut-on sélectionner les embryons bovins avant transfert sur la base de leur métabolisme? La qualité des embryons bovins produits in vitro reste variable. La sélection de ces embryons basée uniquement sur leur morphologie ne permet pas d'atteindre des taux de gestation acceptables. L'utilisation de marqueurs métaboliques pour sélectionner les embryons viables avant transfert apporterait une aide précieuse, tant au niveau économique que comme outil de recherche. Le marqueur idéal doit cependant remplir plusieurs conditions : il doit pouvoir être évalué (1) de manière totalement non invasive ; (2) sur des embryons individuels (ce qui nécessite des techniques très sensibles) ; (3) de manière rapide (pour être compatible avec le transfert frais des embryons) ; (4) il doit permettre de séparer les embryons viables et non viables quel que soit le système de production utilisé. En pratique, ce marqueur n'existe pas, mais certaines techniques d'évaluation du métabolisme s'en rapprochent. La mise au point d'un marqueur métabolique se heurte cependant aux caractéristiques métaboliques de l'embryon, notamment l'évolution du métabolisme au cours du développement embryonnaire et son adaptation aux modifications de l'environnement. (C) Inra/Elsevier, Paris

métabolisme / embryon / bovin / marqueur / in vitro

* Correspondence and reprints

E-mail: donnay@vete.ucl.ac.be 


\section{INTRODUCTION}

Although pregnancy rates after transfer of bovine embryos produced by MOET are now reaching the physiological level, survival rates after transfer of in vitro-produced embryos are still low despite considerable improvement of embryo culture techniques. Moreover, embryo/foetal losses during the first trimester of pregnancy and anomalies in new-borns are more frequent after transfer of in vitro than in vivo embryos. Loss of viability is also greatly increased after cryopreservation of in vitro-produced (IVP) embryos.

Selection of IVP embryos based on morphology as the sole criteria is often poorly correlated with pregnancy rates after transfer. There is thus a need to find markers of embryo viability prior to transfer: 1 ) as a tool to improve in vitro maturation, fertilisation and culture conditions without having to transfer the embryos; 2) to increase pregnancy rates and thus decrease the number of recipient heifers in transfer programmes; 3 ) to increase the commercial value of the selected IVP embryos by decreasing the variability in their quality.

Nutrient uptake has previously been correlated with the developmental capacity after transfer at the blastocyst stage in cattle, mice and humans $[2,6,28,37]$. In retrospective analysis, in vitro glucose uptake by day 10 in vivo-produced bovine blastocysts [28] and day 4 mouse blastocysts [6] was positively correlated with viability after transfer to recipient females. In a follow-up study in mice, Lane and Gardner [17] used both glucose uptake and lactate production (to estimate glycolytic activity - figure 1) to select individual day 4 blastocysts for transfer and reported a four-fold increase in pregnancy rate on this basis.

Several points, however, have to be taken into account before selecting metabolic markers of embryo viability. Some of them will be addressed in this review:

1) the techniques available for measuring embryo metabolism non-invasively;
2) the evolution of metabolism with embryo development;

3) the adaptation of embryo metabolism to its artificial environment;

4) the multiplicity of the roles of metabolic substrates.

\section{NON-INVASIVE TECHNIQUES FOR INVESTIGATING EMBRYO METABOLISM}

The ideal marker of embryo viability should fulfil the following conditions:

1) there should be no alteration of embryo environment (completely non-invasive);

2) be sensitive enough to allow the evaluation of single embryos;

3) easily and rapidly evaluated (allowing for transfer of fresh embryos);

4) highly predictable in all culture conditions (clear cut-off between viable and non-viable embryos).

However, although some techniques for measuring embryo metabolism fulfil some of these conditions, such a marker has not yet been reported.

\subsection{Measurement of uptake/release of glucose, lactate, pyruvate}

Embryos are incubated singly or in small groups in 1- to $4-\mu \mathrm{L}$ droplets of culture medium for a few hours. Afterwards, embryos are removed and the substrate concentrations in the drops after embryo incubation are compared with control drops. The uptake/release by the embryos is then calculated $[8,20]$ (figure 2 ).

The assays for measuring substrate concentrations are based on the generation or consumption of the reduced pyridine nucleotides NADH and NADPH in coupled enzymatic reactions. These nucleotides fluoresce when excited with light at $340 \mathrm{~nm}$, whereas the oxidised forms, $\mathrm{NAD}^{+}$and $\mathrm{NADP}^{+}$do not. 


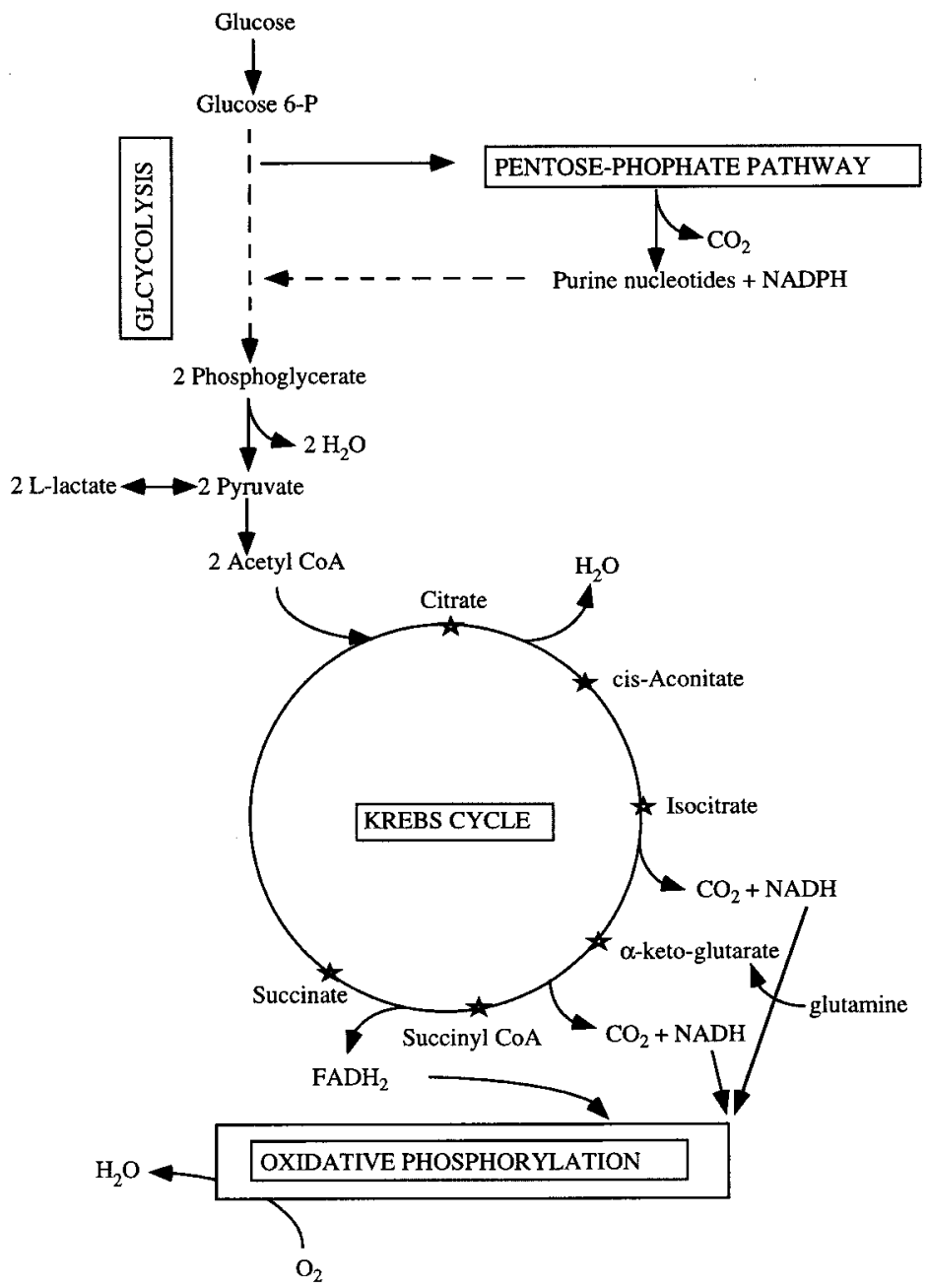

Fig. 1 Pathways of energy metabolism

Figure 1. Pathways of energy metabolism.

Fluorescence is measured directly in small droplets $(10-30 \mathrm{~nL})$ of reactives using a photometer and photomultiplier attached to a fluorescence inverted microscope. Samples (1-3 nL) of spent and control culture medium are taken using previously calibrated home-made capillary pipettes [8]. This miniaturised system allows for the analysis of single embryo metabolism. To evaluate the metabolism of single bovine blas- tocysts, an incubation period of $12 \mathrm{~h}$ in $1-\mu \mathrm{L}$ drops or of $24 \mathrm{~h}$ in $2-\mu \mathrm{L}$ drops is required.

This evaluation is non-invasive; the embryos are cultured in the same medium and gas conditions as for normal culture. The only changes in their environment are 1) the use of fresh medium, and 2) the reduced number of embryos cultured together. This technique has been proven not to interfere with human embryo viability [2]. The main 


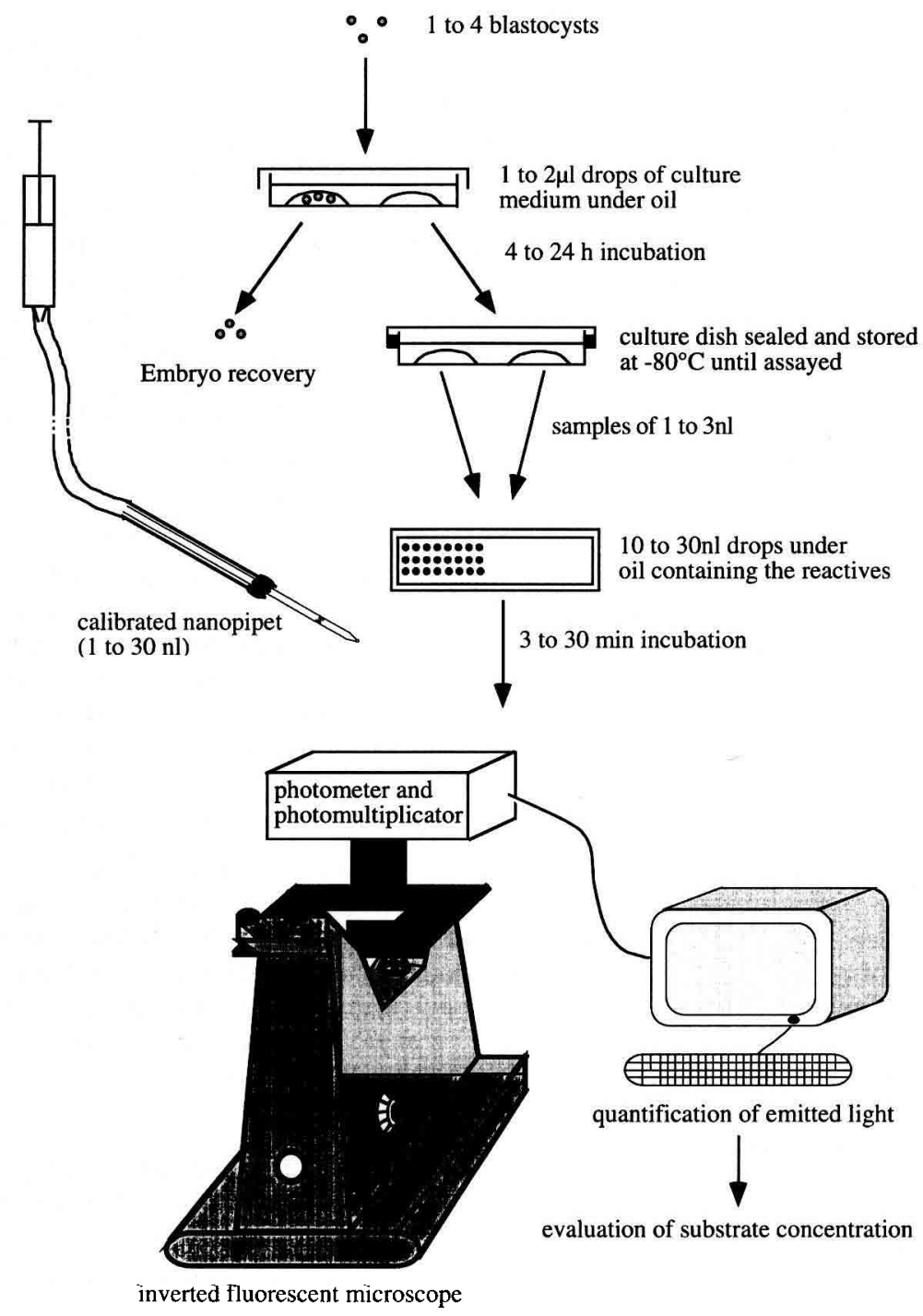

Figure 2. Non-invasive measurement of glucose and pyruvate uptake and lactate release by single embryos.

disadvantages are 1) the delay between the incubation and the evaluation of nutriment uptake/release, which necessitates the freezing of the embryos, and 2) the lack of evaluation of substrate utilisation and metabolic pathways.

Using this technique, Conaghan et al. [2] have observed that pyruvate uptake is lower in embryos from women who became pregnant, compared with those who did not. High uptake could be due to uncoupling of mitochondria, making the inner mitochondrial membrane permeable to protons.

The spent culture drops can also be used to evaluate amino acid uptake/release by reverse-phase HPLC $[3,16,26]$. 


\subsection{Measurement of oxygen uptake}

Several techniques can be used for measuring oxygen uptake, including microelectrodes $[24,25]$ and microspectrophotometry. We describe a simple technique developed in H. Leese's laboratory which involves pyrene, an oil-soluble fluorescent compound, the fluorescence of which is quenched in proportion to the oxygen concentration [13].

Small groups (2-5) of embryos are incubated in a 1- to $2-\mu \mathrm{L}$ volume of Hepesbuffered culture medium in a $5-\mu \mathrm{L}$ PCR micropipette in contact with $1 \mu \mathrm{L}$ of paraffin oil containing pyrene-figure 3 ). The pipette is sealed at both ends and the fluorescence is measured in the oil-phase every 20-30 min for 2-4 h. The increase in fluorescence is proportional to the oxygen uptake of the embryos.

This technique has been shown not to interfere with bovine blastocyst expansion and hatching [3] and could be adapted for the evaluation of single embryos (J.G. Thompson, pers. comm.). Measuring oxygen uptake allows to obtain a direct evaluation of oxidative metabolism from both endogeneous and exogeneous substrates. Another advantage is that the results can be calculated directly and there is thus no need to freeze the embryos. Oxygen uptake, however, might not wholly reflect oxidative phosphorylation, for example when uncoupling of mitochondrial membranes occurs.

This technique was successfully combined with the evaluation of glucose, pyruvate, lactate and amino acid uptake/release on the same embryos and during the same incubation period [3].

\subsection{Evaluation of metabolic pathways using radiolabelled substrates}

This technique described by O'Fallon and Wright [23] was adapted by D. Rieger to the study of single bovine embryos $[29,30]$. It allows for the evaluation of metabolic pathways of several substrates such as glucose, glutamine and pyruvate. Embryos are incubated in the presence of one or two labelled substrates in a drop of culture medium hanging on the cap of a conical tube containing bicarbonate. Radioactive $\mathrm{CO}_{2}$ and $\mathrm{H}_{2} \mathrm{O}$ produced during the metabolism of the labelled substrates are trapped in the bicarbonate solution. After a 3-h incubation period, the embryo is recovered and the radioactivity is evaluated in the bicarbonate solution after addition of $\mathrm{NaOH}$ (to convert the dissolved $\mathrm{CO}_{2}$ and bicarbonate into carbonate) (figure 4).

The main advantage of this technique is that it allows the evaluation of the metabolic pathways of chosen substrates. This technique, however, uses radioactive substrates which could impair further embryonic development. Moreover, it does not allow for the evaluation of the use of endogenous stores such as glycogen, protein and lipids.

Other markers of embryo metabolism on living embryos could include the evaluation of mitochondrial activity using specific probes. The techniques described so far, however, cannot be considered as non-invasive as they involve the addition of the probe to the culture medium and UV irradiation of the embryos.

\section{EVOLUTION OF METABOLISM WITH EMBRYO DEVELOPMENT}

In all mammalian species, preimplantation development can be divided into two phases on the basis of metabolic activity: 1) the period controlled by the mRNAs and proteins accumulated in the oocyte and characterised by a low metabolic activity; and 2) the period following activation of the embryonic genome which shows a dramatic increase in metabolism coinciding with blastocoele cavity formation and expansion [18]. This change in metabolic activity is also associated with the passage of the embryo from the oviduct to the uterus. During the first period, mammalian embryos have a 


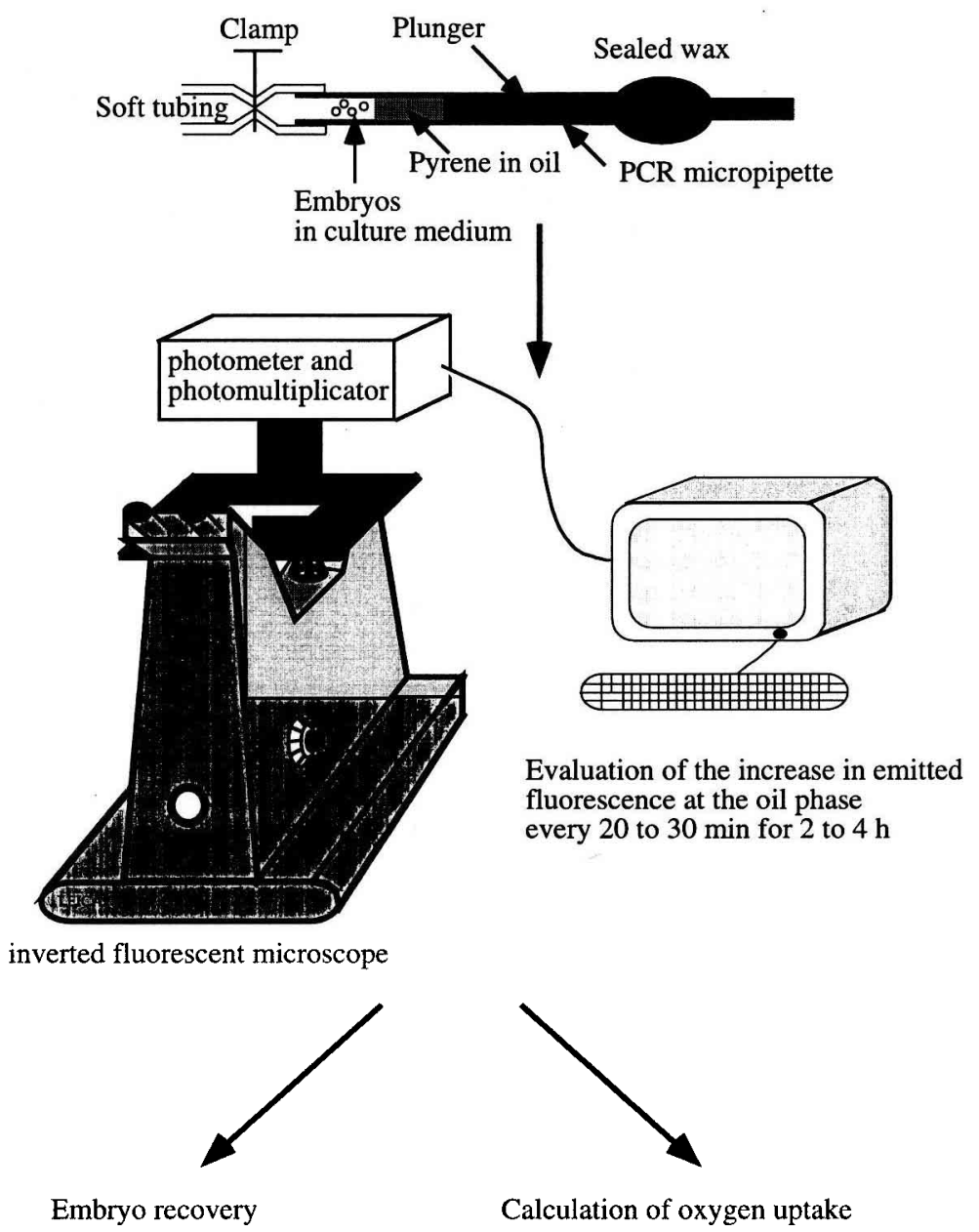

Figure 3. Non-invasive measurement of oxygen uptake by the embryos.

limited capacity to utilise glucose. Pyruvate, lactate and amino acids are the preferred energy substrates [15].

The second period, however, is characterised by an increased use of glucose (by glycolysis) and glutamine. Such a pattern is often observed in rapidly dividing cells where glucose and glutamine are the sources of metabolic intermediates for biosynthetic pathways. For example, glycolysis produces glucose 6-phosphate for the formation of riboses required for DNA and RNA syn- thesis and glycerol 3-phosphate for phospholipid synthesis. Glutaminolysis provides carbon and nitrogen atoms for the biosynthesis of purines, pyrimidines, GTP and $\mathrm{NAD}^{+}[18]$.

Physiological changes in embryo metabolism might thus be associated with an increase in energy demand and/or in biosynthesis but also with modifications in embryo environment, for example in oxygen availability in the uterus before implantation where ATP formation via glycolysis 


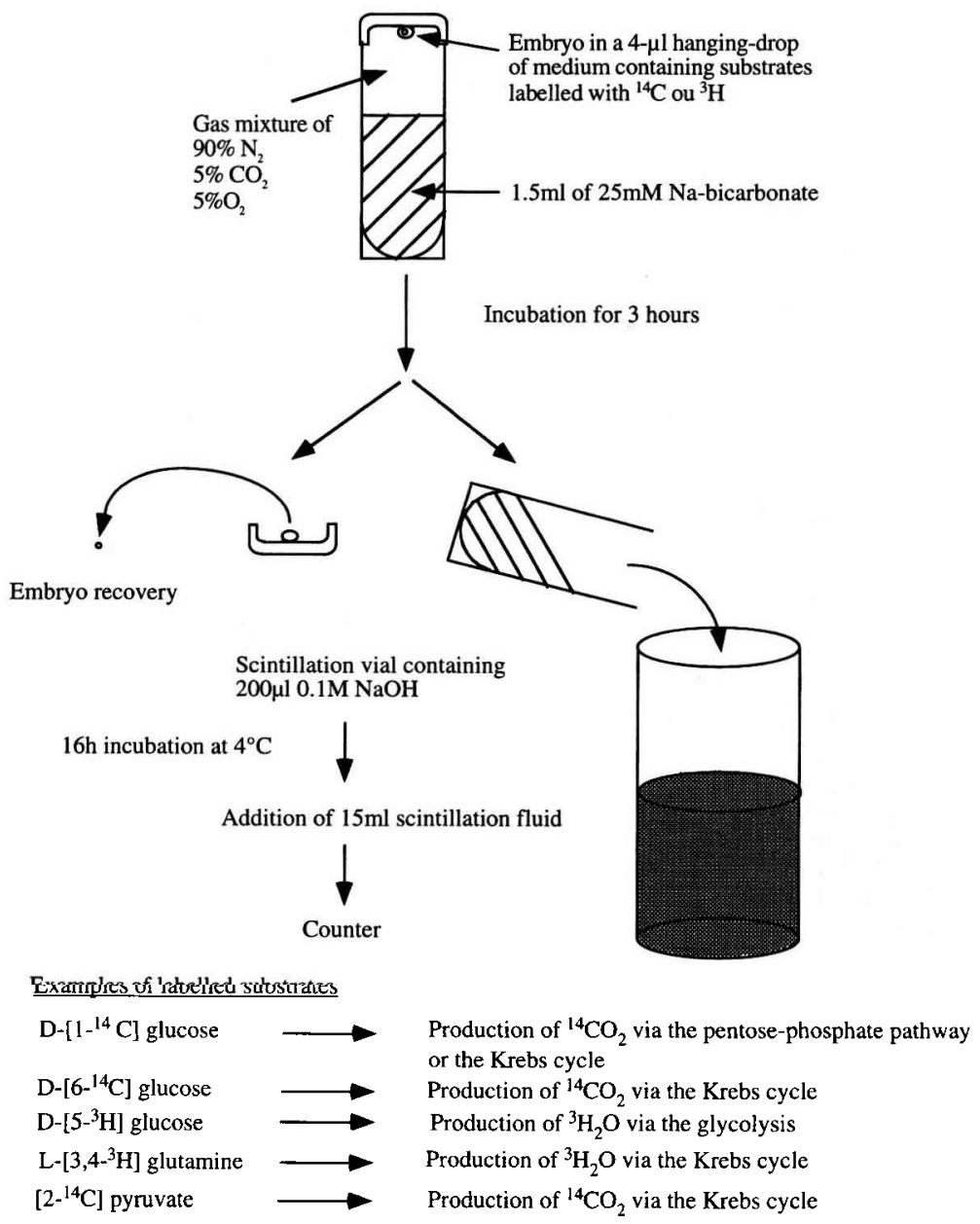

Figure 4. Evaluation of metabolic pathways using labelled substrates.

may be the natural metabolic pathway [19] It is likely that the utilisation of glucose by the early embryo is under the control of specific glycolytic regulators [5]. Inhibitors of glycolysis, such as citrate and EDTA, have a favourable effect on the first stages of embryo development in culture by limiting conversion of glucose to lactate. But they may have adverse effects at the blastocyst stage, namely by reducing the development of the ICM which has been shown to be dependent on glycolysis [12]. Indeed, we also have to keep in mind that the two cell lineages present in the blastocyst show different patterns of energy metabolism as described in the mouse [12].

To assess embryo viability, it is important to evaluate embryo metabolism after the onset of embryonic genome activation: when measurements are performed prior to embryonic genome activation, the observed differences might reflect differences inherited from the oocyte that do not represent the true embryo physiology. In contrast, the 
analysis of nutrient uptake/metabolism at the blastocyst stage will reflect blastocyst function [5].

\section{ADAPTATION OF EMBRYO METABOLISM}

Embryos can adapt their metabolism to their environment. This does not necessarily mean that those metabolic changes are related to a decrease in embryo viability.

\subsection{Comparison between in vitro and in vivo embryos}

Few studies have directly compared the metabolism of in vivo-derived and in vitroproduced embryos. An increase in glycolysis (lactate production) was observed in culture in the mouse [7]. Bovine and ovine embryos produced in vitro convert more glucose to lactate and less to $\mathrm{CO}_{2}[14,34]$. This might be related to an increased incidence of mitochondrial degeneration as observed by Dorland et al. [4] in embryos cultured in the presence of serum, which may alter the oxidative metabolism of such embryos.

In J. Thompson's studies, the rate of ATP production calculated on a per embryo basis seems similar for both in vivo- and in vitroderived bovine blastocysts [33, 35, 36]. Overall, glucose and pyruvate uptake and utilisation as well as oxygen uptake were comparable between in vivo- and in vitroderived embryos, but differed at the cellular level, with increased cellular metabolism for in vitro embryos [34]. Increased oxidative metabolism could in turn induce higher production of reactive oxygen species leading to oxidative stress [21].

Amino acid uptake, however, is higher for in vivo- than for in vitro-produced embryos [26]. This reduced uptake by in vitro-derived blastocysts may represent differences in transport or utilisation of the amino acids.

\subsection{Modifications induced by cryopreservation}

Few data are available on the modification in embryo metabolism induced by cryopreservation. Several kinds of damage are associated with metabolic changes: 1) the death of a proportion of the cells, which decreases the total metabolism on a per embryo basis; 2) the modification in the cell ultrastructure induced by the formation of ice crystals; 3 ) the modifications induced by the toxicity of the cryoprotectants.

A general decrease in both nutrient uptake and utilisation is observed after blastocyst freezing. Both glucose uptake, and to a lesser extent lactate release, could be used to identify, within the first $5 \mathrm{~h}$ after thawing, which blastocyst will continue to develop in vitro and which will fail [9]. There is an increase in oxygen consumption which may be due to damage to the inner membranes of the mitochondria, resulting in partial uncoupling of oxidative phosphorylation. Embryos are able to compensate by increasing glycolytic activity [31].

\section{MULTIPLICITY OF THE ROLES FOR METABOLIC SUBSTRATES}

Some known energy substrates can play several roles that might explain why they are so important in some culture conditions. As a consequence, it may be difficult to use their uptake as a universal marker of viability.

\subsection{The example of pyruvate}

Pyruvate has been shown to be necessary for bovine embryo development in culture. It is taken up by bovine embryos throughout the culture period with a sharp increase at the blastocyst stage [35]. Pyruvate can obviously be used as an energy 
substrate in the Krebs cycle as indicated by the formation of ${ }^{14} \mathrm{CO}_{2}$ from $\left[2-{ }^{14} \mathrm{C}\right]$ pyruvate [30]. But other metabolic characteristics of pyruvate in culture could explain its essential role in vitro.

1) Pyruvate might recycle ammonium ions produced by the non-enzymatic degradation of amino acids and proteins present in the culture medium and by the metabolism of the embryo. In the presence of pyruvate, alanine is released in the embryo culture medium which might indicate that alanine is derived by transamination of pyruvate ( $R$. Partridge and H.J. Leese, unpublished results, figure 5).

2) Pyruvate is also a powerful antioxidant, and has been shown to protect bovine embryos from damage induced by hydrogen peroxides which can be formed during embryo culture [22]. The reaction with hydrogen peroxide produces acetate which can in turn be used as an energy substrate in the bovine [11].

3) Pyruvate may be converted to lactate [1] as a means of controlling intracellular $\mathrm{pH}[10]$.

\subsection{The example of amino acids}

Although glutamine does not support the development of bovine one-cell embryos as the sole substrate in protein-free conditions [27], non-essential amino acids stimulate bovine embryo development during the first

(a)

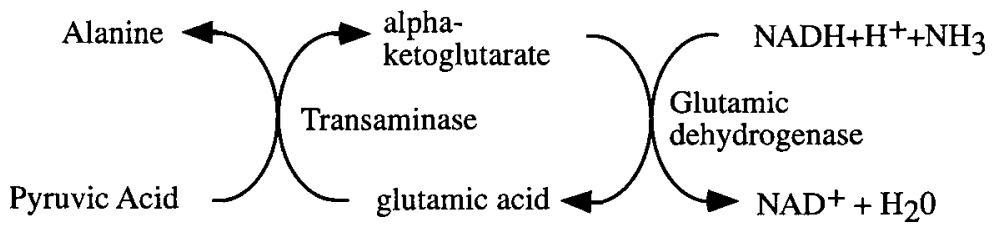

(b)

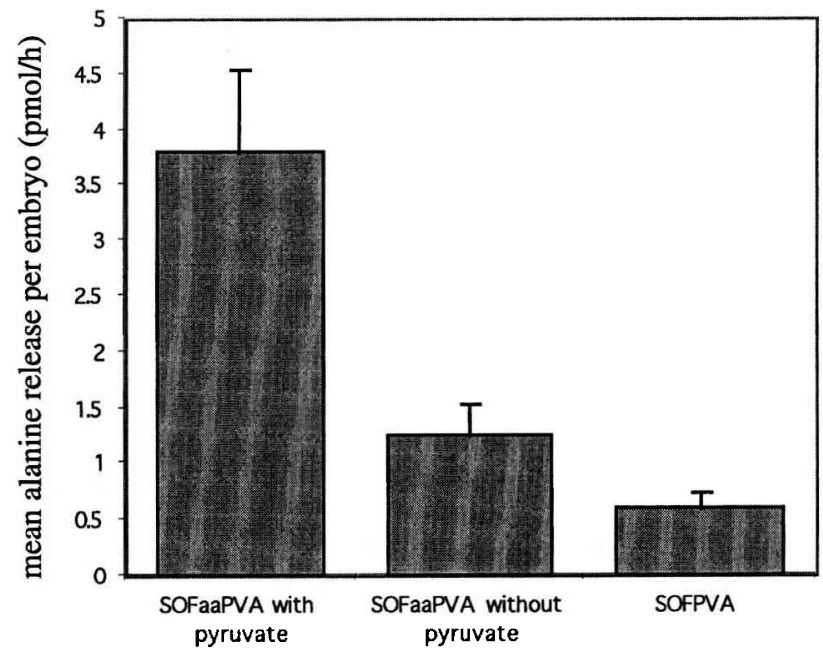

Figure 5. a) Alanine formation from pyruvate and ammonium; b) mean alanine appearance in the medium following culture of bovine blastocysts in the presence and absence of pyruvate and amino acids. 
$72 \mathrm{~h}$ of culture. Afterwards, the highest rates of blastocyst development with the largest number of ICM cells occurred after culture for a further $72 \mathrm{~h}$ in the presence of all 20 amino acids [32]. Several amino acids can enter the Krebs cycle after transformation and be used as energy substrates. Another explanation for their beneficial effect on embryo development may be their ability to regulate the activities of specific energy generating pathways of the embryo, or to act as osmolytes to maintain cell volume, as buffers of $\mathrm{pHi}$ or as antioxidants [5]. The ability of amino acids to minimise culture-induced stress may explain why the presence of amino acids decreases the rate of embryo fragmentation.

\section{CONCLUSIONS}

Embryos can obviously adapt their metabolism to their environment (concentration of energy substrates, oxygen tension). The challenge is to detect when metabolic changes are related to the alteration of embryo development. Another problem is the difficulty in finding predictive factors with a clear cut-off. For example, pyruvate uptake was higher for human embryos that failed to implant than for those which succeeded, but the overlap between the two populations of embryos prevented the selection of embryos for transfer on the basis of pyruvate uptake alone $[2,37]$.

Thus, there is a need to correlate data on embryo metabolism with other markers of cell dysfunction, such as the alteration in mitochondrial integrity, altered kinetics of development, elevated rates of apoptosis and impaired junctional complexes between TE cell. The correlations observed in one culture system will not, however, necessarily exist in another.

In conclusion, given the complexity of embryo metabolism and its adaptability to environmental changes, it is unreasonable to suggest that the measurement of a singu- lar 'key' cellular parameter may be unequivocally adequate to predict the survival of an IVP embryo whatever its culture conditions. A better strategy would be to develop several non-invasive assays, each measuring a specific cellular activity/process within an individual embryo and, if possible, simultaneously [24].

\section{ACKNOWLEDGEMENTS}

This work was supported 'Action de Recherche Concertée n ${ }^{\circ}$ 96/01-196 de la Direction générale de la Recherche scientifique - Communauté Française de Belgique'. R. Partridge and H.J. Leese acknowledge further support from the European Commission and from the UK Ministry of Agriculture, Fisheries and Food.

\section{REFERENCES}

[1] Butcher L., Coates A., Martin K.L., Rotherford A.J., Leese H.J., Metabolism of pyruvate by the early human embryo, Biol. Reprod. 58 (1998) 1054-1056.

[2] Conaghan J., Hardy K., Handyside A.H., Winston R.M.L., Leese H.J., Selection criteria for human embryo transfer: a comparison of pyruvate uptake and morphology, J. Assist. Reprod. Genet. 10 (1993) 21-30.

[3] Donnay I., Leese H., Embryo metabolism during the expansion of the bovine blastocyst, $\mathrm{Mol}$. Reprod. Dev. 53 (1999) 171-178.

[4] Dorland M., Gardner D.K., Trounson A.O., Serum in synthetic oviduct fluid causes mitochondrial degeneration in ovine embryos, J. Reprod. Fertil. Abstract Series 13 (1994) 70.

[5] Gardner D., Changes in requirements and utilization of nutrients during mammalian preimplantation embryo development and their significance in embryo culture, Theriogenology 49 (1998) 83-102.

[6] Gardner D.K., Leese H., Assessment of embryo viability prior to transfer by the non-invasive measurement of glucose uptake, J. Exp. Zool. 242 (1987) 103-105.

[7] Gardner D.K., Leese H.J., Concentrations of nutrients in mouse oviduct fluid and their effects on embryo development and metabolism in vitro, J. Reprod. Fertil. 88 (1990) 361-368.

[8] Gardner D.K., Leese H.J., Assessment of embryo metabolism and viability, in: Trounson A., Gardner D.K. (Eds.), Handbook of in vitro Fertilization, CRC Press, London, 1993, pp. 195-211. 
[9] Gardner D.K., Pawelczynski M., Trounson A.O., Nutrient uptake and utilization can be used to select viable Day 7 bovine blastocysts after cryopreservation, Mol. Reprod. Dev. 44 (1996) $472-475$.

[10] Gibb C.A., Pronnik P., Day M.L., Cook D.I., Control of cytosolic $\mathrm{pH}$ in two-cell mouse embryos: role of $\mathrm{H}+$-lactate cotransport and $\mathrm{Na}+/ \mathrm{H}+$ exchange, Am. J. Physiol. 273 (Cell Physiol. 42) (1997) C404-C419.

[11] Gomez E., Acetate and $\beta$-D-hydroxybutyrate as energy substrates during early bovine embryo development in vitro, Theriogenology 48 (1997) 63-74.

[12] Hewitson L.C., Leese H.J., Energy metabolism of the trophectoderm and inner cell mass of the mouse blastocyst, J. Exp. Zool. 267 (1993) 337-343.

[13] Houghton F.D., Thompson J.G., Kennedy C.J., Leese H.J., Oxygen consumption and energy metabolism of the early mouse embryo, Mol. Reprod. Dev. 44 (1996) 476-485.

[14] Khurana N., Comparative aspects of the energy metabolism of bovine embryos from the oocyte to the hatched blastocyst derived in vitro and in vivo, Ph.D. thesis, Veterinary School, Hannover, Germany, 1992.

[15] Kim J.H., Niwa K., Lim J.M., Okuda K., Effects of phosphate, energy substrates and amino acids on development of in vitro-matured, in vitrofertilized bovine oocytes in a chemically defined protein-free culture medium, Biol. Reprod. 48 (1993) 1320-1325.

[16] Lamb V.K., Leese H.J., Uptake of a mixture of amino acids by mouse blastocysts, J. Reprod. Fertil. 10 (1994) 169-175.

[17] Lane M., Gardner D.K., Prospective selection of viable mouse embryos prior to transfer using metabolic rate, Hum. Reprod. Il (1996) 1975-1978.

[18] Leese H.J., Energy metabolism in preimplantation development, in: Bavister B.D. (Ed.), Preimplantation Embryo Development, Springer-Verlag, New York, 1993, pp. 73-82.

[19] Leese H.J., Metabolic control during preimplantation mammalian development, Hum. Reprod. Update 1 (1995) 63-72.

[20] Leese H.J., Barton A.M., Pyruvate and glucose uptake by mouse ova and preimplantation embryos, J. Reprod. Fertil. 72 (1984) 9-13.

[21] Leese H.J., Donnay I., Thompson J., Human assisted conception: a cautionary tale. Lessons from domestic animals, Hum. Reprod. 13 (Suppl. 4) (1998) 184-202.

[22] Morales H., Tilquin P., Rees J.F., Massip A., Dessy F., Van Langendonckt A., Pyruvate prevents peroxide-induced injury of in vitro preimplantation bovine embryo, Mol. Reprod. Dev. 52 (1999) 149-157.

[23] O'Fallon J.V., Wright R.W. Jr, Quantitative determination of the pentose phosphate path- way in preimplantation mouse embryos, Biol. Reprod. 34 (1986) 58-64.

[24] Overstrom E.W., Embryo respiration as an objective measure of embryo quality, Proceedings of the Satellite Workshop on Embryo development in vitro, Current challenges and future concepts, International Embryo Transfer Society, Boston, 1998, pp. 22-23.

[25] Overstrom E.W., Duby R.T., Dobrinsky J., Roche J.F., Boland M.P., Viability and oxidative metabolism of the bovine blastocyst, Theriogenology 37 (1992) 269

[26] Partridge R.J., Leese H.J., Consumption of amino acids by bovine preimplantation embryos, Reprod. Fertil. Dev. 8 (1996) 945-950.

[27] Pinyopummintr T., Bavister B.D., Energy substrate requirements for in vitro development of early cleavage-stage bovine embryos, Mol. Reprod. Dev. 44 (1996) 193-199.

[28] Renard J.P., Philippon A., Menezo Y., In-vitro uptake of glucose by bovine blastocysts, J. Reprod. Fertil. 58 (1980) 161-164.

[29] Rieger D., Guay P., Measurement of the metabolism of energy substrates in individual bovine blastocysts, J. Reprod. Fertil. 83 (1988) 585-591.

[30] Rieger D., Loskutoff N.M., Betteridge K.J., Developmentally related changes in the uptake and metabolism of glucose, glutamine and pyruvate by cattle embryos produced in vitro, Reprod. Fertil. Dev. 4 (1992) 547-557.

[31] Rieger D., Pollard J.W., Leibo S.P., The effect of cryopreservation on the metabolic activity of in vitro produced cattle blastocyst, Cryobiology 30 (1993) 631

[32] Steeves T.E., Gardner D.K., Temporal effects of amino acids on bovine embryo development in culture, Biol. Reprod. 57 (Suppl. 1) (1997) 25.

[33] Thompson J.G., Comparison between in vivoderived and in vitro-produced pre-elongation embryos from domestic ruminants, Reprod. Fertil. Dev. 9 (1997) 341-354.

[34] Thompson J.G., Simpson A.C., Pugh P.A., Wright R.W. Jr, Tervit H.R., Glucose utilization by sheep embryos derived in vivo or in vitro, Reprod. Fertil. Dev. 3 (1991) 571-576.

[35] Thompson J.G., Partridge R.J., Houghton F.D., Cox C.I., Leese H.J., Oxygen uptake and carbohydrate metabolism by in vitro derived bovine embryos, J. Reprod. Fertil. 106 (1996) 299-306.

[36] Thompson J.G., Partridge R.J., Houghton F.D., Kennedy C.J., Pullar D., Leese H.J., Oxygen consumption by Day 7 bovine blastocysts: determination of ATP production, Anim. Reprod. Sci. 43 (1996) 241-247.

[37] Turner K., Martin K.L., Woodward B.J., Lenton E.A., Leese H.J., Comparison of pyruvate uptake by embryos derived from conception and non-conception natural cycles, Hum. Reprod. 9 (1994) 2362-2366. 